\title{
A Distinção Contável-Massivo e a Expressão de Número no Sistema Nominal
}

\author{
(The Count-Mass Distinction and the Expression of \\ Number within Nominal Systems)
}

\author{
Nize Paraguassu-Martins \\ (USP - Universidade de São Paulo) \\ Ana MülLER \\ (USP - Universidade de São Paulo)
}

\begin{abstract}
This work investigates the count-mass distinction within natural language nominal systems. In particular, we focus on the count-mass distinction and its relation to number morphology. We claim that there is no necessary typological correlation between the lack of number marking and the nonexistence of a count-mass distinction for common nouns. Joosten's (2002) proposal is presented with the purpose of showing that the count-mass distinction can be treated either as a lexical distinction among the denotations of common nouns, or as a distinction among Noun Phrase constituents.
\end{abstract}

KEY-WORDS: semantics; mass nouns; count nouns; number.

RESUMO: Este trabalho investiga a distinção contável-massivo entre os nomes comuns. Em particular, nos debruçamos sobre a distinção contável-massivo e sua relação com a morfologia de número. Defendemos que não existe uma correspondência tipológica necessária entre a ausência de marcação de número e a inexistência de uma distinção entre nomes contáveis e nomes massivos. Além disso, a classificação das abordagens da distinção contável-massivo proposta por Joosten (2002) é apresentada com a finalidade de mostrar que essa distinção pode ser tratada ou como uma distinção lexical entre as denotações dos nomes comuns ou como uma distinção entre tipos de sintagmas nominais. PALAVRAS-CHAVE: semântica; nomes massivos; nomes contáveis; número.

D.E.L.T.A., 23:esp., 2007 (65-83) 


\section{Introdução}

Neste trabalho, tratamos da distinção lingüística entre nomes contáveis e nomes massivos e de sua relação com a morfologia de número.

O texto está organizado em duas seções. A seção 1 trata das diferentes abordagens para a distinção contável-massivo nas línguas naturais. A seção mostra que a distinção pode ser tratada como uma distinção lexical entre as denotações dos nomes comuns ou pode ser tratada como uma distinção entre tipos de sintagmas nominais. Já a seção 2 discute a relação entre a expressão da distinção contável-massivo e a expressão da noção de número (singular vs. plural) nas línguas naturais. Essa discussão nos mostra que não existe uma correspondência tipológica necessária entre a ausência de marcação de número e a inexistência de uma distinção entre nomes contáveis e nomes massivos.

\section{Abordagens da distinção contável-massivo}

Tradicionalmente, nomes comuns são subdivididos em contáveis e massivos. Intuitivamente, a diferença está em que nomes contáveis se referem a entidades conceitualizadas como discretas e nomes massivos se referem a entidades conceitualizadas como contínuas. Quine apresenta claramente essa intuição:

To learn 'apple' it is not sufficient to learn how much of what goes on counts as apple; we must learn how much counts as an apple, and how much as another. Such terms possess built-in modes, however arbitrary, of dividing their reference... Water is scattered in discrete pools and glassfuls... still it is just 'pool', 'glassful', ..., not 'water'... that divide their reference (Quine 1960:91). ${ }^{1}$

Essa divisão se manifesta gramaticalmente em várias línguas. Vamos ilustrá-la com algumas propriedades do inglês, que é a língua mais exaus-

\footnotetext{
1 'Para aprender o que expressa 'maçã' não é suficiente aprender que quantidade do que ocorre conta como maçã; nós precisamos aprender que quantidade conta como uma maçã, que quantidade conta como outra. Tais termos possuem modos internos, mesmo que arbitrários, de dividir sua referência... Água está espalhada em poças e copos... ainda assim é apenas 'poça', 'copo', ... não 'água'... que divide sua referência” (Quine 1960:91, tradução nossa - Paragussu-Martins \&Müller).
} 
tivamente discutida pela literatura sobre o assunto. ${ }^{2}$ No inglês, nomes contáveis possuem uma forma plural e uma forma singular, como ilustrado pelas sentenças em (1). Uma outra característica dos nomes contáveis é a de poderem ser diretamente combinados com numerais. Essa propriedade também é ilustrada pelas sentenças em (1).

(1) a. There is one apple on the table lá está uma maçã sobre a mesa 'Tem uma maçã na mesa'

b. There are two apples on the table lá estão duas maçãs sobre a mesa 'Tem duas maçãs sobre a mesa'

Já nomes massivos não podem ser diretamente contados, como ilustram as sentenças em (2), e necessitam de classificadores ou de sintagmas de medida para serem apropriadamente contados, como se pode ver pelo contraste entre a agramaticalidade de (2a-b) e a gramaticalidade de (3a) com a inserção de expressão de medida bars 'barras'. Nomes massivos também não aceitam a marcação de plural, como se pode ver em (3b).

(2) a. *There is one gold on the table lá está um ouro sobre a mesa

b. *There are two golds on the table lá estão dois ouros sobre a mesa

(3) a. There are two bars of gold on the table lá estão duas barras de ouro sobre a mesa 'Tem duas barras de ouro na mesa'

b. *There are two bars of golds on the table lá estão duas barras de ouros sobre a mesa

Nomes contáveis podem ser ordenados (ver sentença (4a)), ao passo que nomes massivos não são ordenáveis (ver sentença (4b)).

(4) a. My second apple was fantastic minha segunda maçã estava fantástica 'Minha segunda maçã estava fantástica'

\footnotetext{
2 Como o português brasileiro coloquial aceita expressões como 'três banana' e 'duas água' e 'banana faz bem para a saúde' optamos por não utilizar essa língua para ilustrar a distinção gramatical entre nomes massivos e contáveis.
} 


\section{b. *My second gold was fantastic meu segundo ouro foi fantástico}

A distinção entre nomes contáveis e massivos foi introduzida na lingüistica por Jespersen (1924). O autor, utilizando-se de critérios semânticos, chama de 'substantivos contáveis' os nomes que transmitem uma idéia de coisa definida, com formato e limites precisos, como seriam, por exemplo, livro, carro, canção, etc. e de 'substantivos massivos' os nomes que não apresentam tais características, como por exemplo, prata, amor, terra, etc; qualquer dos tipos pode ser concreto ou abstrato. ${ }^{3}$

Muitos lingüistas vieram a questionar a caracterização de Jespersen, pois o fato de os nomes transmitirem ou não idéia de coisa definida, com formato e limites precisos, não é um critério confiável para se estabelecer a distinção lingüística entre nomes contáveis e massivos (cf. Ware 1979 e Bunt 1985, entre outros). Há nomes que são contáveis e não transmitem idéia de coisa definida, como sonho, virtude, crença, entidade, etc. e nomes que são massivos e transmitem idéia de coisa definida, como mobilia, verdura, etc.

A literatura sobre a questão ainda não chegou a um consenso sobre em que termos uma teoria sobre a distinção contável-massivo deve ser definida, se em termos sintáticos, semânticos, ontológicos ou contextuais. Lingüistas e filósofos muitas vezes não são explícitos sobre os critérios utilizados para efetuar tal distinção (sobre essa questão, ver Pelletier (1979)). Assim, vale a pena explicitar e analisar os diferentes critérios sobre os quais tal classificação se baseia.

Joosten (2002) classifica a literatura existente sobre a distinção contável-massivo em quatro abordagens: gramatical, ontológica, semântico-conceptual e contextual. Abaixo segue a descrição de cada uma delas.

\subsection{Abordagem gramatical}

Para a abordagem gramatical, há, no léxico, nomes contáveis e massivos, mas tal distinção só é visível em termos sintáticos. O que existe para

Material e immaterial, no original. 
essa abordagem é uma sintaxe para os nomes contáveis e outra para os nomes massivos. Por exemplo, em inglês, os quantificadores many/few ('muitos'/poucos') só selecionam nomes contáveis e os quantificadores much/ little ('muito/pouco'), só selecionam nomes massivos. Observe as construções abaixo:

(5) a. *much book/many books muito livro muitos livros

b. much wine/ *many wines ${ }^{4}$ muito vinho muitos vinhos

'Muito vinho'

Para a abordagem gramatical, sabemos que o nome book 'livro', em (5a), é contável não porque este possui uma denotação atômica, mas porque é selecionado por many 'muitos'. Da mesma forma, sabemos que wine 'vinho', em (5b), é massivo porque é selecionado por um much 'muito' e não por many 'muitos'. A abordagem gramatical ignora a relação entre propriedades sintáticas e semânticas para estabelecer a distinção contávelmassivo.

Outra propriedade sintática que caracteriza a distinção entre nomes contáveis e massivos é que, na presença de numerais cardinais, nomes massivos exigem a presença de classificadores ou de sintagmas de medida. ${ }^{5}$ Observe as sentenças abaixo:

(6) a. John bought two books John comprou dois livros 'John comprou dois livros'

b. John bought $*$ two silvers/ grams of silver John comprou duas pratas/gramas de prata

'John comprou duas pratas/duas gramas de prata'

\footnotetext{
4 Many wines é gramatical numa leitura taxionômica significa 'muitos tipos de vinho', que não é a leitura em questão no dado citado.

5 A literatura não é clara sobre a definição de classificadores. Há quem distinga classificadores de sintagmas de medida, argumentando que os primeiros dizem respeito a propriedades inerentes aos objetos referidos, ao passo que os segundos são responsáveis por criar unidades abstratas de contagem, independentemente da existência "ontológica" de tais unidades (cf. Avelar 2005, Chierchia 1998, Lyons 1977). Neste trabalho, não se discutirá essa questão. Assumiremos, com Doetjes (1997), que classificadores são nomes capazes de nomear ou criar unidades de medida aos nomes que com eles ocorrem. Em (6b), grams 'gramas' é um classificador porque cria a unidade de medida para o nome silver 'prata'.
} 
Em (6a), books 'livros' é um nome contável porque não necessita de um classificador ou sintagma de contagem diante do numeral cardinal two 'dois'. Por outro lado, em (6b), o nome silver 'prata' é um nome massivo porque, para que seja interpretado como quantidade da substância exige um classificador, no caso grams 'gramas', diante do numeral cardinal two 'dois' (cf. Lyons 1977, Gillon 1992, entre outros).

No entanto, a abordagem gramatical apresenta alguns problemas. Em primeiro lugar, ela não explica os casos em que nomes contáveis são empregados como massivos, como em (7), e os casos em que nomes massivos são empregados como contáveis, como em (8):

(7) The house is built of brick a casa é construída de tijolo

'A casa é feita de tijolo'

(8) John bought excellent wines John comprou excelentes vinhos 'John comprou vinhos excelentes'

Em (7), brick 'tijolo' não apresenta marca morfossintática de número, característica sintática própria dos nomes massivos no inglês, enquanto que, em (8), wines 'vinhos' apresenta tal marca, característica sintática própria dos nomes contáveis.

Em segundo lugar, a abordagem gramatical não explica a tendência de nomes contáveis denotarem entidades atômicas e de nomes massivos denotarem entidades não atômicas. Parece improvável que seja puramente coincidência tal tendência!

\subsection{Abordagem ontológica}

Reflexões ontológicas sobre a distinção contável-massivo são freqüentes na literatura. Para a abordagem ontológica, há no léxico uma distinção entre nomes contáveis e massivos, mas tal distinção é operada entre entidades do mundo real, isto é, são as propriedades do mundo real que determinam se um nome é ou não contável. 
Para essa abordagem, nomes massivos são nomes que se referem a entidades com as seguintes propriedades no mundo: cumulatividade, distributividade e homogeneidade. Se um elemento possui a propriedade da cumulatividade, a soma de elementos de mesma natureza resulta em um elemento de natureza igual. Por exemplo, no inglês, wine 'vinho' é um nome massivo porque se no mundo real adicionarmos vinho a vinho teremos como resultado vinho (cf. Quine 1960).

Se um conjunto possui a propriedade da distributividade, suas partes conservam a mesma natureza do conjunto todo. Por exemplo, no inglês, wine 'vinho' é um nome massivo porque se no mundo real removermos uma porção de vinho de um todo, o resultado será sempre vinho (cf. Cheng 1973).

Por fim, a propriedade da homogeneidade está relacionada aos nomes que se referem a entidades com certa estrutura interna tal que sua divisão em objetos atômicos, distintos entre si, não é possível. Por exemplo, no inglês, wine 'vinho' é um nome massivo porque possui uma estrutura homogênea, isto é, partes de vinho são todas semelhantes e podem ser chamadas de vinho (cf. ter Meulen 1981).

Os nomes contáveis, segundo a abordagem ontológica, são nomes que se referem a entidades atômicas, com formas bem definidas e composição heterogênea, como os nomes 'anel' e 'garrafa'. Nomes como esses possuem referência atômica e composição heterogênea porque suas denotações não se aplicam a nenhuma de suas partes. Por exemplo, as partes de um anel são diferentes e não podem ser chamadas de anel, da mesma forma que as partes de uma garrafa não podem ser chamadas de garrafa. Ou, inversamente, um anel não pode ser dividido em anéis, assim como uma garrafa não pode ser dividida em garrafas.

No entanto, essa abordagem apresenta problemas. Primeiramente, se a distinção contável-massivo dos nomes é uma propriedade do mundo real, espera-se que não haja diferentes escolhas entre as diferentes línguas. Contudo, isso nem sempre se verifica. Em inglês, hair 'cabelo' é massivo enquanto que, no francês, cheveux 'cabelos' é contável. Em português 'fruta' é contável, mas a contraparte em inglês (fruit) é massivo.

Em segundo lugar, a abordagem ontológica pode apenas ser bem sucedida se aplicada a nomes concretos, embora seja evidente que a distinção 
contável-massivo também se aplica aos nomes abstratos, como se pode ver pelo contraste entre (9a) e (9b).

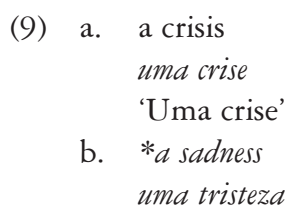

Em terceiro lugar, a abordagem ontológica envolve temas não lingüísticos. Ela tende a cair em uma discussão que tem pouco a ver com a língua, como por exemplo, até que ponto se pode dividir um referente em suas partes mínimas. Ou seja, qual seria o tamanho mínimo que as partes do referente de um nome $\mathrm{X}$ precisam ter para ainda poderem ser concebidas como X (cf. Novais 1992).

\subsection{Abordagem semântico-conceptual}

Para a abordagem semântico-conceptual, a distinção entre nomes contáveis e massivos não está relacionada com as entidades do mundo real, mas com a maneira como cada língua percebe e conceitualiza essas entidades, se contínua ou descontinuamente. Em outras palavras, para essa abordagem a distinção contável-massivo reside no próprio conceito mental a que os nomes correspondem e não nas coisas que eles nomeiam.

Segundo essa abordagem, nomes contáveis e massivos são diferentes no que diz respeito à maneira como cada um conceitualiza a realidade. Nomes contáveis conceitualizam a realidade em termos de entidades atômicas e nomes massivos, em termos de entidades não atômicas. Observe as sentenças em (10) e (11).

(10) John bought a car

John comprou um carro

'John comprou um carro'

(11) *John bought a wine ${ }^{6}$

John comprou um vinho

6 A sentença só é gramatical se houver um classificador implícito, como 'garrafa' ou 'copo', por exemplo. 
Em (10), podemos dizer a car porque o nome car 'carro' é conceitualizado como sendo uma entidade bem definida com propriedades específicas aplicáveis apenas à entidade como um todo. Por outro lado, em (11), como o nome wine 'vinho' é conceitualizado como uma entidade sem partes bem definidas e propriedades específicas aplicáveis a todas as partes, não é possível referir-se diretamente a uma entidade denotada por esse nome. Essa propriedade é percebida através da possibilidade ou não de ocorrência do artigo.

A abordagem semântico-conceptual está relacionada com a abordagem gramatical, mas não necessariamente com a abordagem ontológica. Em inglês, furniture 'mobília', por exemplo, é ontologicamente um nome contável, pois se refere a entidades discretas. ${ }^{7}$ Para a abordagem gramatical furniture 'mobília' é um nome massivo porque, apesar de fazer referência a entidades discretas do mundo real, como chairs 'cadeiras', tables 'mesas', etc., é um nome selecionado apenas por contextos sintáticos massivos, como pode ser ilustrado em (12):

(12) *a furniture /much furniture /*many furnitures uma mobilia muita mobília muitas mobílias 'Muita mobília'

Já para a abordagem semântico-conceptual, a massividade de furniture 'mobília' deve-se a uma reorganização conceptual do mundo: objetos separados são conceitualizados de forma homogênea. A diferença entre os objetos que fazem parte da denotação de furniture 'mobília' é escondida por não ser relevante para a transmissão da mensagem em particular.

Um problema para esta abordagem é que é pouco provável que todos os desvios da distinção contável-massivo dos nomes possam ser explicados em termos de conceitualização. Por exemplo, a abordagem semântico-conceptual não explica porque, em inglês, pea 'ervilha' é um nome contável e rice 'arroz' é um nome massivo.

\footnotetext{
7 Nomes como furniture são considerados pela literatura como pertencentes a um gênero de coletivos cuja característica principal é a superordenação a conjuntos de lexemas numa relação hierárquica ambivalente quanto à distinção entre a hiponímia e a relação de parte-todo. Sobre essa discussão, ver Lyons (1977)
} 


\subsection{Abordagem contextual}

Para a abordagem contextual, a distinção contável-massivo não é uma característica do nome, mas do sintagma em que o nome está inserido. Observe as sentenças em (13) e (14):

(13) John bought wine John comprou vinho 'John comprou vinho'

(14) John bought two excellent wines John comprou dois excelentes vinhos 'John comprou dois vinhos excelentes'

Em (13), a ausência de determinante e de morfologia de número significa conceitualização não-individualizada e, por isso, o nome wine 'vinho' é interpretado como uma leitura massiva, não individualizada. Em (14), a presença do determinante two 'dois' significa conceitualização individualizada; dessa forma o nome wine 'vinho' é interpretado com uma leitura contável, que pode ser ou uma leitura taxionômica, onde estão sendo contabilizados tipos de vinho, ou uma leitura onde a informação do que contar como unidade é fornecida pelo contexto.

A distinção contável-massivo, segundo a abordagem contextual, está relacionada em primeiro lugar à marcação de contabilidade. Para essa abordagem, a contabilidade é um fenômeno lingüístico associado ao sintagma e não às entradas lexicais dos nomes (cf. Allan 1980). A distinção contávelmassivo não seria uma distinção operada entre nomes, mas entre ocorrências de nomes, sendo, portanto, determinada pelos quantificadores e determinantes que são apropriados aos nomes (cf. Ware 1979, Bunt 1985). A idéia básica dos defensores dessa abordagem é que os nomes podem ser usados tanto como contáveis quanto como massivos.

Mas como todas as outras abordagens, a abordagem contextual também apresenta um problema, que é o de não explicar por que a maioria dos nomes obviamente favorece um tipo de contexto em detrimento de outro. 


\subsection{Sintese e proposta}

Se aplicamos os critérios utilizados pelas diferentes abordagens discutidas acima a alguns dados do português brasileiro, percebemos a necessidade de repensar essa classificação com base no tipo de objeto lingüístico utilizado por cada abordagem. Observe as sentenças abaixo:

(15) João comprou duas canetas

(16) João comprou duas garrafas de vinho

Em (15), o nome 'caneta' pode ser classificado como um nome contável porque possui conceitualização atômica e, conseqüentemente, unidade natural de contabilidade (abordagem semântica). Nomes como 'caneta' referem-se a entidades atômicas no mundo (abordagem ontológica) e quando submetidos a operações de contabilidade não necessitam de um classificador (abordagem gramatical). Por outro lado, em (16), 'vinho' pode ser classificado como um nome massivo, porque não possui conceitualização atômica e, conseqüentemente, unidade natural de contabilidade (abordagem semântica). Nomes como 'vinho' referem-se a entidades não atômicas (abordagem ontológica) e quando submetidos a operações de contabilidade necessitam de um classificador ou de um sintagma de medida, no caso, 'garrafa' (abordagem gramatical).

A breve discussão acima mostra que as abordagens semântico-conceptual, ontológica e gramatical têm em comum o fato de que todas se aplicam aos nomes comuns. Por outro lado, a abordagem contextual da distinção contável-massivo, diferentemente das abordagens anteriormente mencionadas, não se aplica aos nomes comuns, mas ao sintagma nominal. Para essa abordagem, o que existe são contextos contáveis e massivos nos quais quaisquer nomes podem ser inseridos. Isso porque ela está relacionada com a contabilidade do sintagma nominal: contextos com marcação de contabilidade são contextos que tornam o sintagma nominal contável e contextos sem marcação de contabilidade são contextos que tornam o sintagma nominal massivo.

Analisando as sentenças (15) e (16), sob a luz da abordagem contextual, temos que tanto o sintagma nominal 'duas canetas', em (15), quanto o sintagma nominal 'duas garrafas de vinho', em (16), são contáveis, pois 
independentemente das denotações que possuem seus núcleos estão em contextos sintáticos que tornam o sintagma nominal contável.

De acordo com o exposto acima, vemos que as abordagens gramatical, ontológica e semântico-contextual são abordagens lexicais. Temos, portanto, apenas dois tipos de abordagens: a lexical e a contextual. As abordagens gramatical, ontológica e semântico-conceptual são abordagens lexicais porque se baseiam em evidências de que o léxico de muitas línguas humanas faz uma distinção entre nomes comuns contáveis e massivos. Por outro lado, a abordagem contextual trata da denotação do sintagma nominal no que diz respeito à sua contabilidade.

Essa sistematização dos tipos de abordagem possibilita a separação entre a noção de denotação de um nome comum e a noção de contabilidade de um sintagma nominal. Esta separação é importante para explicar os dados de línguas em que, diferentemente do inglês, a denotação de um nome comum e sua contabilidade não coincidem em todos os contextos, como é o caso do português brasileiro. Observe as sentenças abaixo:

(17) João vende livro

(18) João vende água

Segundo qualquer uma das abordagens lexicais, 'livro' seria um nome contável e 'água' seria um nome massivo. No entanto, no contexto das sentenças (17) e (18), nenhum dos dois sintagmas é contável, pois o contexto em que ocorrem não diz nada sobre como está organizada sua denotação. Os livros, por exemplo, podem estar sendo vendidos tanto como volumes individuais, como por quilo para reciclagem.

Vemos então que a distinção contável-massivo enquanto um fenômeno lexical deve ser analisada à parte da distinção contável-massivo enquanto um fenômeno contextual.

A partir deste ponto, vamos assumir a possibilidade de existência de uma distinção lexical entre nomes massivos e contáveis no léxico das línguas naturais. ${ }^{8}$ No entanto, não vamos nos posicionar sobre qual das abor-

Para uma discussão detalhada sobre a questão ver Paraguassu \& Müller (2006). 
dagens - a gramatical, a ontológica ou a conceitual - seria a "correta", pois a discussão passa de certa forma por uma discussão entre a relação linguagem-mundo e extrapola os limites deste artigo.

$\mathrm{Na}$ próxima seção, passamos à discussão de o que significa ter uma denotação massiva ou contável e se existe uma relação entre o tipo de denotação de um nome e a possibilidade de esse nome expressar singularidade ou pluralidade.

\section{Criando indivíduos: número e a distinção contável-massivo}

Link (1983) propõe que as denotações de nomes massivos e dos nomes contáveis pertencem a domínios ontológicos diferentes. Segue, portanto, a intuição básica de que a linguagem descreve o mundo como contendo dois tipos de entidades distintas: (i) entidades discretas ou atômicas e (ii) matéria indiferenciada ou substância.

Uma vez assumida essa ontologia, a flexão de número é interpretada como uma operação sobre o domínio das entidades discretas, pois apenas nesse caso a distinção entre entidades atômicas (unidades) e entidades plurais (grupos formados por duas ou mais unidades) faz sentido. Explica-se assim porque apenas nomes contáveis podem ser pluralizados.

Nomes comuns contáveis são analisados pelo autor como denotando conjuntos de indivíduos atômicos. ${ }^{9}$ A denotação de um nome comum singular como 'porco' é o conjunto de todos os porcos, o que está ilustrado em (19). Nomes comuns plurais, por outro lado, são descritos como denotando tanto indivíduos atômicos quanto indivíduos plurais, ou seja, conjuntos de mais de um indivíduo, como ilustrado em (20) para o nome contável plural 'porcos'.

9 Na verdade, nomes comuns são analisados pela semântica formal como funções de mundos possíveis a conjuntos de indivíduos. Neste artigo, vamos nos ater estritamente a uma descrição extensional das denotações. Não faremos uso de denotações intensionais. Isto é, não trabalharemos com funções que tomam mundos possíveis como argumentos (ver Chierchia 2003 e Oliveira 2000, entre outros, sobre a diferença entre extensão e intensão e sobre a denotação dos nomes comuns). 
(19) $[$ [porco $]]=\left\{\right.$ porco $\left._{\mathrm{a}}, \operatorname{porco}_{\mathrm{b}}, \operatorname{porco}_{\mathrm{c}}, \ldots\right\}$

(20) $[$ [porcos $]\}=\left\{\right.$ porco $_{\mathrm{a}}, \operatorname{porco}_{\mathrm{b}}, \operatorname{porco}_{\mathrm{c}}, \ldots,\left\{\operatorname{porco}_{\mathrm{a}}, \operatorname{porco}_{\mathrm{b}}\right\},\left\{\operatorname{porco}_{\mathrm{b}}\right.$, $\left.\operatorname{porco}_{c}\right\},\left\{\operatorname{porco}_{a}, \operatorname{porco}_{c}\right\}, \ldots,\left\{\right.$ porco $\left.\left._{a}, \operatorname{porco}_{b}, \operatorname{porco}_{c}\right\}, \ldots\right\}$

O tratamento semântico dos nomes comuns descrito acima pode ser chamado de clássico e é a posição tradicionalmente apresentada pelos manuais de introdução à semântica formal. ${ }^{10}$ Ele se estabeleceu com base no comportamento da maior parte das línguas românicas e germânicas. Nessas línguas, nomes singulares não podem ocorrer em contextos semanticamente plurais. Nomes plurais, por outro lado, podem ocorrer tanto em contextos plurais como em contextos neutros.

Em inglês, por exemplo, nomes contáveis singulares podem ocorrer apenas em contextos singulares. $O$ contraste de gramaticalidade entre (21) e (22) ilustra esse fato. A explicação corrente é que a sentença (22) é agramatical porque o significado do nome comum singular boar 'javali' entra em choque com o significado plural do numeral two 'dois'. Nomes contáveis plurais, por outro lado, podem ocorrer tanto em contextos plurais, como em contextos neutros em relação ao número de entidades denotadas. A sentença (23) mostra que um nome contável singular em inglês não pode expressar neutralidade em relação a número, enquanto um nome contável plural como o da sentença (24) está apto a fazer isso. Note-se que a sentença (24) será verdadeira tanto se João comprou apenas uma quanto se comprou duas ou mais maçãs.

(21) John killed one boar John matou um javali 'John matou um javali'

(22) *John killed two boar João matou dois javali

(23) *John bought apple John comprou maçã

(24) John bought apples John comprou maçãs 'João comprou maçãs'

10 Ver, por exemplo, Chierchia (2000), de Schwart (1998) e Portner (2005). 
No que diz respeito aos nomes massivos, Link propõe que a extensão desses nomes é representada por um conjunto contendo toda a substância do mundo contida na denotação desse nome e por subconjuntos contendo porções dessa substância, que se dividem em novas partes sem que nunca sejam obtidos átomos, como representado em (25):

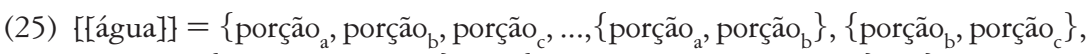
$\left\{\right.$ porçãa $_{a}$, porção $\left._{c}\right\}, \ldots,\left\{\right.$ porção $_{a}$, porçãa $_{b}$, porção $\left.\left._{c}\right\}, \ldots\right\}$

Existem, entretanto, línguas como o chinês, o japonês e o tailandês, que não possuem flexão de número (ou esta é opcional) e, ao mesmo tempo, possuem classificadores cuja presença é obrigatória em contextos de contagem. Em chinês, por exemplo, a presença de um classificador é exigida tanto para a contagem de um nome aparentemente contável como $b i$ ('caneta'), como para a contagem de um nome aparentemente massivo como rou ('carne'). Observe o contraste de gramaticalidade causado pela presença de classificadores nas expressões em (26a) e (27a) e pela sua ausência nas expressões em (26b) e (27b).

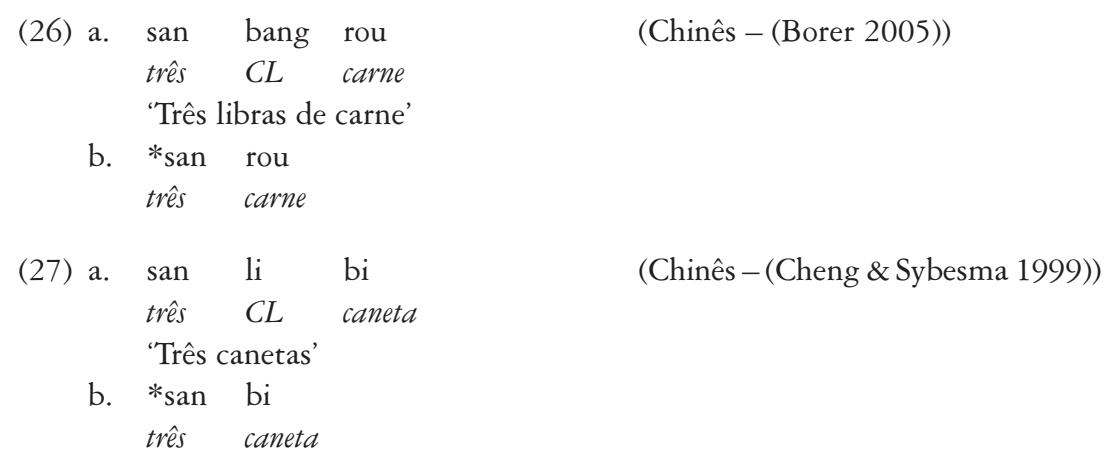

Línguas classificadoras têm sido analisadas pela literatura como línguas nas quais os nomes comuns são todos massivos (ver Chierchia 1998a e Krifka 1995, entre outros). Nessas línguas não haveria distinção entre nomes massivos e nomes contáveis. Nelas, a função dos classificadores seria a de transformar a denotação massiva dos nomes comuns em uma denotação composta por entidades atômicas e, conseqüentemente, tornar esses nomes passíveis de contagem.

No entanto, quando se examina uma maior variedade de línguas, a aparente dicotomia entre classificadores e flexão de número não se susten- 
ta. Existem línguas como o Yágua - língua da família Peba-Yágua, falada no Noroeste do Peru - e o Totonac - língua da família Totonac-Tepehua, falada em Sierra Norte, México - que possuem tanto classificadores como flexão de número. Os exemplos (28)-(30) mostram a existência de flexão de número em Yágua. Já (31) ilustra o uso de classificadores em contextos de contagem.

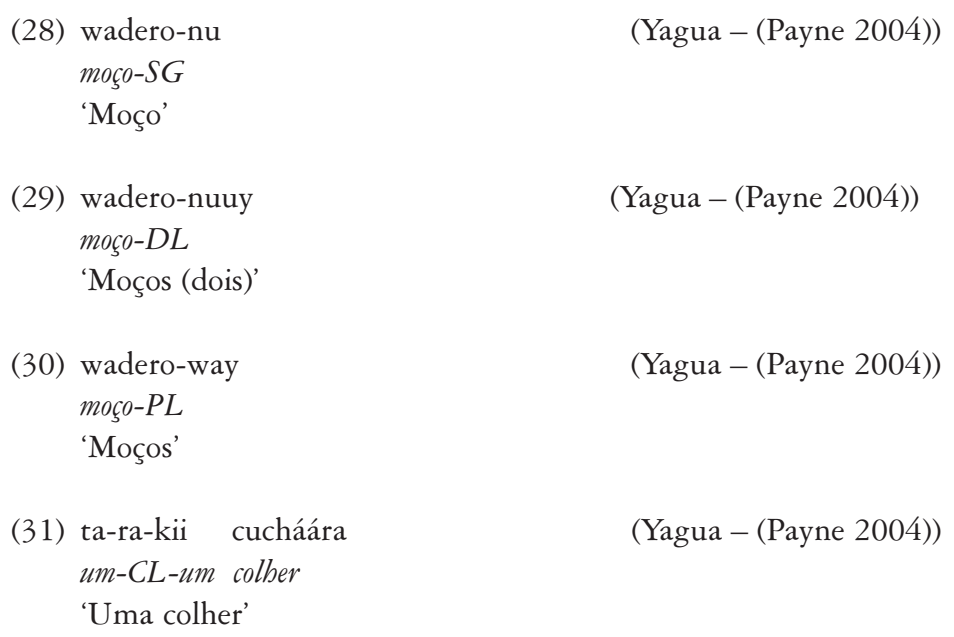

Existem também línguas nas quais a flexão de número é opcional. Línguas como húngaro e turco não fazem uso de classificadores ou de flexão de número para contagem e, no entanto, possuem flexão de singularplural. A sentença (32) mostra a existência de marcação de pluralidade em húngaro. Já a sentença (33) mostra que nomes podem ser contados sem o uso de flexão nessa língua.

$\begin{array}{ll}\text { (32) Mari verseket olvas } & \text { (Húngaro-(Rullmann 2003)) } \\ \text { Maria poema.PL.ACC lêe } & \\ \text { 'Maria lê poemas' } & \\ \text { (33) ot hajo } & \text { (Húngaro - (Rullmann 2003)) } \\ \text { cinco navio } & \\ \text { 'Cinco navios' } & \end{array}$

Por outro lado, alguns trabalhos têm argumentado a favor da existência da distinção massivo-contável tanto em línguas classificadoras (ver Cheng \& Sybesma 1999 e Doetjes 1997 sobre o chinês) como em línguas que não 
possuem morfologia de número (ver Wilhelm 2005 para o Dëne Suliné e Müller et al. 2006 para o Karitiana). Doetjes (1997) apresenta como uma das evidências a favor da existência dessa distinção no chinês o fato de o classificador geral ge 'unidade' só ser possível com nomes contáveis como podemos ver contrastando (34) com (35). Esses trabalhos põem em questão a existência de uma correlação necessária entre a presença de classificadores e a presença de uma denotação massiva.
(34) san ge shu
(Chinês - (Doetjes (1997))
três $C L$ livro
'Três livros'
(35) * san ge rou
três $C L$ carne
(Chinês - (Doetjes (1997))

Vemos então que não se pode afirmar que ausência de morfologia de número em uma determinada língua significa necessariamente que nessa língua todos os nomes possuem denotações lexicais massivas. Vimos também que um sintagma não precisa estar pluralizado para ser contabilizado.

\section{Sumário}

A classificação de Joosten (2002) da literatura sobre a distinção contável-massivo pode ser agrupada em duas abordagens: abordagens lexicais e abordagem contextual. A primeira trata da denotação contável-massivo dos nomes comuns e a segunda, da denotação contável-incontável dos sintagmas nominais.

A distinção lexical contável-massivo nas línguas naturais não coincide necessariamente com a possibilidade ou não de contabilidade no sintagma. Vimos também que a ausência de morfologia de número em uma língua natural não implica que essa língua não possua uma distinção lexical entre nomes massivos e contáveis.

$$
\begin{array}{r}
\text { E-mails: } \\
\text { anamuler@usp.br } \\
\text { nparaguassu@yahoo.com.br }
\end{array}
$$




\section{REFERÊNCIAS BibliográficAs}

Avelar, M. 2005. Relatório científico FAPESP. Ms., Universidade de São Paulo.

Allan, K. 1980. Nouns and countability. Language 56: 541-567.

Borer, H. 2005. Structuring sense. Oxford: Oxford University Press.

Bunt, H. 1985. Mass terms and model-theoretic semantics. Cambridge: CUP. Cheng C-Y. 1973. Comments on Moravcsik's paper. In: K. J. J. HintikkA, J. M. E. Moravcsik \& P. Suppes (orgs.) Approaches to natural language. Dordrecht: D. Reidel. 286-288.

Cheng, L. \& R. Sybesma. 1999. Bare and not-so-bare nouns and the structure of NP. Linguistic Inquiry 30: 509-542.

Chierchia, G. 1998. Plurality of mass nouns and the notion of 'semantic parameter' In: S. RothSTEIN (org.) Events and grammar. Dordrecht: Kluwer Academic Publishers. 10-53. . 2003. Semântica. Campinas: Editora da UNICAMP.

DoETJES, J. 1997. Quantifiers and selection. On the distribution of quantifying expressions in French, Dutch and English. PhD Dissertation, Leiden University. The Hague: HAG.

Gillon, B. 1992. Towards a common semantics for English count and mass nouns. Linguistics and Philosophy 15: 597-639.

Joosten, F. 2002. Account of the count/mass distinction: A critical survey. Ms., FWO-Vlaanderen/KU Leuven, Belgium. [Disponível em KULeuven/FWOVlaanderen:http://wwwling.arts. kuleuven.ac.be/ nedling/fjoosten/scl2hand.htm]

JesPersen, O. 1924. The philosophy of grammar. London: George Allen and Unwin Ltd.

KRIFKA, M. 1995. Common nouns: A contrastive analysis of Chinese and English. In: G. Carlson \& F. Pelletier (orgs.) The generic book. University of Chicago Press. 398-411.

LINK, G. 1983. The logical analysis of plurals and mass terms: a latticetheoretical approach. In: R. BAEUERLE et al. (org.). Meaning, use and interpretation of language. Berlin, New York: de Gruyter. 302-323.

Lyons, J. 1977. Semantics. Cambrigde: Cambridge University Press.

ter Meulen, A. 1981. An intensional logic for mass terms. Philosophical Studies 40: 105-125.

Müller, A., L. Storto \& T. Coutinho-Silva. 2006. Número e a distinção contável-massivo em karitiana. Revista da ABRALIN 5: 185-213. 
NovaIs, M. C. 1992. Aspectos da referência massiva. Cadernos de Semântica. Lisboa: Faculdade de Letras da Universidade de Lisboa.1-32.

Oliveira, R. P. 2000. Semântica formal: Uma breve introdução. Campinas: Mercado de Letras.

Paraguassu, N. \& A. Müller. 2006. The count-mass distinction in natural languages. Trabalho apresentado no VI Workshop em Lingüística Teórica. UFSC: Florianópolis.

PaYne, D. 2004. The source and function of Yagua classifiers. Ms., University of Oregon.

Pelletier, F. 1979. Mass terms: Some philosophical problems. Dordrecht: Reidel.

PORTNER. P. 2005. What is meaning? Fundamentals of formal semantics. Blackwell.

QUINE, W. 1960. Word and object. Cambridge Massachusetts: MIT PRESS. Rullmann, H. \& A. Yo. 2003. General number and the semantics and pragmatics of indefinite bare nouns in Mandarin Chinese. Ms., University of Calgary, Canada. [Disponível em http://semanticsarchive.net/Archive/ jhlZTY3Y/]

de Swart, H. 1998. Introduction to natural language semantics. Stanford: CSLI Publications.

Ware, R. 1979. Some bits and pieces. In. F. Pelletier (org.) Mass terms: Some philosophical problems. Dordrecht: Reidel

WiLHelm, A. 2005. Bare nouns in Dëne Suliné. Talk presented at SULA-3, Buffalo, USA. 